\title{
Proposal for an academic Association for Medical Humanities
}

Robert Arnott, Gillie Bolton, Martyn Evans, Ilora Finlay, Jane Macnaughton, Richard Meakin, William Reid

Following an initiative taken by the Nuffield Trust, an ad hoc steering committee consisting of the authors of this paper was convened under the auspices of the Trust in order to bring about the establishment of an academic Association for the Medical Humanities in the United Kingdom and Ireland (see page 63).

The present paper seeks to do two things: (A) to propose the founding of an academic Association for Medical Humanities, as a representative focus for medical humanities research and teaching in medical schools and universities in the United Kingdom and Ireland; and (B) to propose for discussion a conception of the nature, identity and scope of medical humanities as a field of study.

\section{(A) An Association for Medical}

\section{Humanities}

1. Several universities and associated medical schools in the British Isles have introduced, or are preparing to introduce, the medical humanities as a field of academic teaching and/or research.

2. The medical humanities needs a national, specific, academic association, comparable to those enjoyed by most if not all academic disciplines, to enable those engaged in the field to identify and discuss matters of common interest and to share their experiences regarding teaching and research.

3. The Association for Medical Humanities is proposed as a voluntary academic association to play such a role.

4. The Association for Medical Humanities should facilitate the development of both teaching and research in the medical humanities, by means of regular meetings and conferences where academic, professional and administrative matters can be discussed in person, subsequently to be pursued and disseminated through publications including the Medical Humanities journal (to which the association will affiliate) as well as through e-mail discussion lists, etc.

5. The association will complement, rather than be identified with, organisations representing practitioners of "arts in health".

6. In terms of teaching, the association's concerns will specifically include:

6.1 helping to develop educational materials in the medical humanities
6.2 helping to develop curricula appropriate to medical humanities teaching in different contexts

6.3 establishing recognition of medical humanities in relation to "bench-marking" in medical education as well as comparable "benchmarking" within relevant humanities disciplines

6.4 promoting the reputation, within higher education, of medical humanities as a serious field of academic learning and teaching

6.5 optimising the teaching resources nationally available to medical humanities

6.6 facilitating the pooling and/or exchange of teaching resources and educational materials in medical humanities among participating higher education institutions

6.7 encouraging resource allocation for this discipline within medical schools

7. In terms of research, the association's concerns will specifically include:

7.1 promoting shared agendas for medical humanities research

7.2 encouraging, and where appropriate initiating, specific research collaborations

7.3 promoting the recognition of medical humanities as a serious area of academic research within the remit of private and public funding bodies including the Arts and Humanities Research Board (AHRB), the Economic and Social Research Council (ESRC) and the Medical Research Council (MRC)

7.4 identifying and promoting specific research themes by means of regular academic conferences

7.5 optimising the research resources nationally available to the medical humanities

7.6 encouraging and promoting international research and educational collaboration

7.7 promoting connections with the arts and health field while recognising the unique roles of each field

\section{(B) The medical humanities as a field of inquiry \\ INTRODUCTION}

"The medical humanities" is, in the United Kingdom, a relatively new term for a sustained interdisciplinary inquiry into aspects of medical practice, education and research expressly con- 
cerned with the human side of medicine. These are, most especially, the nature, importance and role of human experience on the part of patients and practitioners alike, including their experience of the patient-practitioner relationship.

Historically the first and most obvious feature of this inquiry was the modern exploration of medical ethics. "The medical humanities" is the name of a more inclusive inquiry, though one that embraces ethics.

SCOPE

The humanities generally can be said to be concerned with the recording and interpretation of human experience. They are typically taken to include the study of history, literature, philosophy, ethics, anthropology, sociology, theology, psychology, social psychology (and, on some readings, law).

The medical humanities are therefore those same disciplines expressly concerned with the human experiences of health, disease, illness, medicine and health care.

\section{INTERDISCIPLINARITY}

The medical humanities should be viewed as integral to medicine (ie as constitutive of our understanding of medicine's nature and goals, alongside the medical sciences) rather than as a series of optional extras to an essentially scientific conception of medicine.

The medical humanities are therefore interdisciplinary rather than simply multidisciplinary. This means that the contributing disciplines attempt to understand and share one another's perspectives and metaphors-and, ultimately, that humanities and sciences also attempt this with regard to each others' conceptions of medicine.

\section{'INTERSUBJECTIVE' KNOWLEDGE}

Patients have detailed knowledge of their own experiences of illness. Doctors have detailed scientific knowledge of disease processes. These two kinds of knowledge appear very different, and bringing them together is not straightforward. If done successfully, then both patient and doctor have a shared understanding which could be said to be "intersubjective" knowledge.

This is a difficult thing to achieve, and clinical practitioners are less well prepared for it than they are for simply bringing to bear their scientific knowledge. But clinical method obviously relies on the interpretation of patients' subjective experiences as well as on scientific data.

The types of enquiries and the methods used in the medical humanities can help us to understand and value this "inter-subjective" knowledge.

ACTIVITY

The medical humanities involve the engagement of the perspectives and inquiries characteristic of the humanities and social sciences with the nature, goals and knowledge-base of medical education, teaching and research. They also involve sustained reflection on that engagement.
This is quite distinct from undertaking arts projects in the health arena. The therapeutic use of the arts is one of the central phenomena which the medical humanities should try to understand and describe (as well as applaud); but the role of understanding and describing such phenomena is wholly distinct from engaging in them as practitioners.

AIMS

The medical humanities might reasonably pursue most or all of the following aims:

- to undertake and promote interdisciplinary research into the human side of medicine

- to emphasise education as distinct from training in an appropriately university-based medical curriculum, and to emphasise the importance of curiosity in learning

- to contribute to the development of students' and practitioners' abilities to listen, interpret and communicate, and to encourage their sensitive appreciation of the ethical dimensions of practice

- to stimulate and encourage a fitting and enduring sense of wonder at embodied human nature

- to stimulate and encourage a spirited sense of educational inquiry

- to encourage students and practitioners to identify, explore, develop and sustain their own professional and personal values

- to develop students' and practitioners' skills in thinking critically and reflectively about their experience and knowledge

- to encourage the development of insights into, and concern for, different aspects of the human condition

- to enable students and practitioners to view their own experiences in a broader humanistic and historical context

- to encourage an acceptance amongst students and practitioners that medicine is neither primarily a scientific nor primarily an arts based discipline but integrates understandings which grow out of both "cultures"

- to promote a wider access into medicine from students with educational backgrounds other than the sciences.

Robert Arnott is Director, Centre for the History of Medicine, University of Birmingham Medical School; Gillie Bolton is Research Fellow, School of Health and Related Research, University of Sheffield; Martyn Evans is Senior Lecturer, School of Health Science, University of Wales Swansea; Baroness Finlay of Llandaff is Vice-Dean of Medicine, University of Wales College of Medicine; Fane Macnaughton is Director, Centre for Arts and Humanities in Health and Medicine, University of Durham; Richard Meakin is Co-Director, Centre for Medical Humanities, Royal Free and University College Medical School, London; Sir William Reid is the former Parliamentary Ombudsman for Health, Edinburgh.

\section{References}

1 Declaration of Windsor. In: Philipp R, Baum M, Mawson A, Kalman KC. The humanities in medicine: beyond the millennium. London: The Nuffield Trust, 1999. 